\title{
Changes in weight following peroral endoscopic myotomy: results from a North American center
}

\section{다(1) $(\odot)$}

\section{Authors}

Simon Hew ${ }^{1,2}$, Douglas Motomura², Robert Bechara ${ }^{2}$

Institutions

1 Monash Medical Centre Clayton, Gastroenterology and Hepatology, Clayton, VIC, AUS

2 Queen's University, Department of Medicine, Kingston, ON, CAN

submitted 9.12.2020

accepted after revision 19.5.2021

\section{Bibliography}

Endosc Int Open 2021; 09: E1491-E1496

DOI 10.1055/a-1520-0016

ISSN 2364-3722

(C) 2021. The Author(s).

This is an open access article published by Thieme under the terms of the Creative Commons Attribution-NonDerivative-NonCommercial License, permitting copying and reproduction so long as the original work is given appropriate credit. Contents may not be used for commercial purposes, or adapted, remixed, transformed or built upon. (https://creativecommons.org/licenses/by-nc-nd/4.0/)

Georg Thieme Verlag KG, Rüdigerstraße 14,

70469 Stuttgart, Germany

Corresponding author

Simon Hew, Monash Medical Centre Clayton, Gastroenterology and Hepatology, 246 Clayton Road, Clayton, Victoria, Australia

simonhew@hotmail.com

\section{ABSTRACT}

Background and study aims Weight loss is a feature of untreated achalasia. While weight gain after achalasia treatment is expected, little data exist about the extent of it, particularly after peroral endoscopic myotomy (POEM). We aimed to describe the changes in weight after POEM.

Patients and methods A retrospective analysis was performed of patients from March 2016 to March 2020 undergoing POEM by an experienced operator at an academic referral center in Kingston, Ontario, Canada. Data examined included pre- and post-POEM body mass index (BMI), procedural characteristics, and Eckardt scores.

Results Ninety-two patients underwent POEM during the study period, for 77 of whom follow-up data were available including BMI. Prior to POEM, the median BMI was 26. Six patients $(7.8 \%)$ were considered to be underweight, 24 (31.2\%) were normal, 26 (33.8\%) were pre-obese, and 21 (27.2\%) were obese. At a median follow-up of 15 months, the clinical success rate was $95 \%$. Patients in the normal weight and pre-obesity categories had a significant increase in weight post-POEM. Of the underweight patients, two $(33.3 \%)$ moved up to a normal weight category and two $(33.3 \%)$ became pre-obese. In the normal BMI category, eight $(37.5 \%)$ became pre-obese and one patient (4.2\%) became obese. Ten of the 26 pre-obese patients (38.4\%) were obese post-POEM. Seven of the obese patients (33.3\%) reached Class III (morbid) obesity at followup.

Conclusions Patients may experience a marked increase in BMI following POEM, with over one-third of patients becoming pre-obese, obese or morbidly obese at follow-up in our study in a North American population. Further study is required to analyze weight gain patterns post-POEM.

\section{Introduction}

Achalasia is an esophageal motility disorder characterized by the dysfunction of smooth muscle relaxation of the lower esophageal sphincter (LES) and abnormal esophageal peristalsis. In North America, it has an estimated prevalence of 10 per 100000 [1]. Achalasia results in dysphagia and regurgitation and can be accompanied by chest pain. As the condition persists, weight loss can ensue.

There are a number of treatment strategies for achalasia. Pneumatic balloon dilation of LES and surgical Heller's myotomy have been the mainstay of treatment for several decades. With the advancement of endoscopic tools and techniques facilitating access to the submucosal space and beyond, there now exists an endoscopic therapy that allows for precise myot- 
omy of the LES. Reported by Inoue in 2010 [2], peroral endoscopic myotomy (POEM) is a safe and effective treatment for all variants of achalasia. The clinical success of POEM recently has been reported as up to $98 \%$ at 12 months [3], with durability seen up to 5 years after treatment [4]. POEM is readily becoming the treatment modality of choice for achalasia in some centers.

At present, there is a paucity of data regarding changes in weight after successful achalasia treatment, particularly after POEM. In treated achalasia, reversal of weight loss, or weight gain, should be expected. Because achalasia can affect individuals of all weight classes, and perhaps is more common in those that are already pre-obese or obese [5], successful treatment may inadvertently lead to excessive weight gain.

The aim of this study was to describe the changes in weight in patients that had undergone POEM in a North American center.

\section{Patients and methods}

\section{Patients}

All patients undergoing POEM between March 2016 and March 2020 at an academic referral center in Canada (Kington Health Sciences Center, Kingston, Ontario) were included. Achalasia was defined on the basis of manometric, endoscopic and radiographic findings, with subtypes established on the basis of high-resolution manometry as per the Chicago Classification v3.0 [6]. Patient information was recorded in a database. Preprocedural characteristics such as age, diagnosis, baseline weight and height, body mass index (BMI), metabolic risk factors, prior treatments, and baseline Eckardt score were included. After discussion, local Institutional Review Board approval was waived due to the retrospective nature of the study.

\section{POEM}

All POEM procedures were performed by one experienced endoscopist (RB) who had performed over 350 cases as an assistant or partial operator and over 60 cases as the primary operator before the study period. POEM was performed under general anesthesia and with endotracheal intubation in the operating theater. After esophageal cleaning and definition of the anatomy, submucosal injection in the 5 o'clock position (posterior approach) was performed. Using the triangle tip knife (KD-640 L or KD-645L; Olympus, Tokyo, Japan), a mucosal incision was performed then a submucosal tunnel was created and extended to just beyond the gastroesophageal junction. A selective circular muscle myotomy was performed. The mucosal incision was closed with clips. Technical success was determined by the successful completion of the myotomy. Patients were admitted for observation overnight and had a repeat esophago-gastroduodenoscopy (EGD) the next day to assess the integrity of the mucosa. If the mucosa was intact, patients were started on clear fluids and discharged home (Day 1 ).

On Day 2, patients could advance their diet to pureed foods, followed by a soft diet on Day 3. On Day 4, a regular diet could be started. Prophylactic oral antibiotics, sucralfate, and twicedaily proton pump inhibitors were also prescribed on discharge.
No formal nutrition or dietary advice was provided as part of routine pre- or post-procedural care.

Patients were recalled for a clinical follow-up 3 to 4 months after POEM. This consisted of an EGD, high-resolution esophageal manometry, 24-hour $\mathrm{pH}$ study, and barium swallow. Patients were offered EGD every 1 to 3 years thereafter, primarily to assess for endoscopic features of recurrent achalasia, reflux esophagitis, and surveillance for esophageal squamous dysplasia. BMI was calculated and recorded at the most recent followup appointment. Clinical success was defined by as an Eckardt score $<3$ [7]. The Eckard score is a tool that assesses dysphagia, regurgitation, chest pain and weight loss. Patients were excluded from analysis if there were no follow-up data on BMI available.

\section{Statistical analysis}

Patients were analyzed according to their BMI at time of referral for POEM. The World Health Organization categories for BMI were used to divide patients into underweight (below 18.5), normal ( 18.5 to 24.9 ), pre-obesity (25.0 to 29.9 ), obesity class I (30.0 to 34.9 ), obesity class II (35.0 to 39.9 ), and obesity class III (above 40). Categorical values were presented as number (\%) and continuous variables were expressed as median and interquartile range (IQR). Difference in medians for paired data was analyzed with the sign test given non-parametric distribution. Difference in medians across multiple groups was analyzed with Mood's median test. Categorical differences were analyzed via Pearson's chi-square test. Statistical significance was set at $P<0.05$. Statistical analysis was performed using SPSS (Version 26; SPSS Inc, Chicago, Illinois, United States).

\section{Results}

A total of 92 patients underwent POEM during the study period, with 77 patients $(83.7 \%$ ) having follow-up at least 3 months after treatment. Patient characteristics are presented in $>\mathbf{T a}$ ble 1. The median age was 55 years and the majority were male (54.5\%). Type II achalasia was the most common subtype. Median duration of symptoms before diagnosis was 60 months (IQR 24-120 months). The median pre-POEM Eckardt score was 7 (IQR 6-9).

- Table 2 presents patient characteristics for each BMI category. At baseline, the median BMI was 26 . Six patients $(7.8 \%)$ were underweight, 24 (31.2\%) were normal, 26 (33.8\%) were pre-obese, and 21 (27.3\%) were obese. Of the obese patients, eight (38.1\%) had Class I obesity, 10 (47.6\%) had Class II obesity and three (14.3\%) had Class III obesity. Sixteen patients ( $21 \%)$ had at least one metabolic risk factor of diabetes, dyslipidemia or hypertension, and seven patients (9\%) had all three risk factors. There was a similar distribution of metabolic risk factors between the weight categories.

POEM was a technical success in $100 \%$ of patients. With a median follow-up duration of 15 months (interquartile range 11-28.5 months), 73 patients (95\%) had achieved clinical success. The median post-POEM Eckardt score was 1 (IQR 0-2). Post-POEM reflux, using the Lyon Consensus definition [8], was present in 19 of 51 patients (37.2\%) in whom 24-hour pH test- 
- Table 1 Patient characteristics and POEM outcomes.

\begin{tabular}{|c|c|}
\hline & $N=77$ \\
\hline Males & $42(55 \%)$ \\
\hline Age (years) & $55(32.5-68)$ \\
\hline \multicolumn{2}{|l|}{ Achalasia (Chicago Classification) } \\
\hline - Type 1 & $14(18 \%)$ \\
\hline - Type 2 & $39(51 \%)$ \\
\hline - Type 3 & $22(29 \%)$ \\
\hline " Other & $2(3 \%)$ \\
\hline \multicolumn{2}{|l|}{ Metabolic comorbidities } \\
\hline - Diabetes & $10(13 \%)$ \\
\hline - Dyslipidemia & $18(23 \%)$ \\
\hline - Hypertension & $27(35 \%)$ \\
\hline \multicolumn{2}{|l|}{ Comorbidity burden } \\
\hline - None of above & $45(58 \%)$ \\
\hline - 1 of above & $16(21 \%)$ \\
\hline - 2 of above & $9(12 \%)$ \\
\hline - 3 of above & $7(9 \%)$ \\
\hline Duration of symptoms (months) & $60(24-120)$ \\
\hline Pre-procedure Eckardt & $7(6-9)$ \\
\hline Myotomy length (cm) & $17(14-20)$ \\
\hline Post-procedure Eckardt & $1.0(0-2)$ \\
\hline Clinical success & $73(95 \%)$ \\
\hline Post-POEM reflux (Lyon Consensus) & $19(37 \%)$ \\
\hline Pre-procedure BMI & $26(21.8-30.1)$ \\
\hline Post-procedure BMI & $27.5(24.5-32.9)$ \\
\hline Follow-up duration (months) & $15(11-28.5)$ \\
\hline
\end{tabular}

ing had been completed. There was no significant difference in rates of post-POEM reflux between patients that increased BMI category $(37.9 \%)$ and those that did not $(34.8 \%, P=0.06)$.

- Table 3 details the change in BMI post-POEM. Overall, there was a median increase in BMI from 26.8 to 29.1. Patients in the normal weight and pre-obesity categories had a significant increase in weight post-POEM ( $>$ Fig. 1 ). $>$ Table 4 details the change in BMI categories. Of the underweight patients, two $(33.3 \%)$ moved up to a normal weight category and two (33.3\%) became pre-obese. In the normal BMI category, eight (33.3\%) became pre-obese and one patient (4.2\%) became obese. Ten of the 26 pre-obese patients (38.4\%) were obese postPOEM. Seven of the obese patients $33.3 \%$ ) reached Class III (morbid) obesity at follow-up. Overall, 28 patients $(36.3 \%$ ) moved up a BMI category and an additional 12 patients (15.6\%) were pre-obese or obese after POEM. No patients in the pre-obese or obese categories attained a normal BMI at follow-up. There were no significant differences in follow-up duration between the BMI categories $(P=0.85)$.

\section{Discussion}

Our study examined the changes in BMI after POEM for achalasia. In 77 patients, $61 \%$ of whom were considered to be pre-obese or obese at baseline. After a technically and clinically successful POEM, at a median follow-up of 15 months, there was a significant increase in weight for patients in the normal weight and pre-obesity categories. There were an additional $15.6 \%$ of patients who had become pre-obese or obese. In patients who were either pre-obese or obese pre-POEM, over one-third became obese or morbidly obese, respectively, on follow-up.

Some weight gain is expected after achalasia treatment. As achalasia progresses and with longer symptom duration, pathologic weight loss ensues. There is evidence that even in obese patients, untreated achalasia is correlated with a risk of malnutrition [5]. Part of successful treatment is the recovery of this weight loss. Reduction in LES pressure and esophageal dysmotility after myotomy is intended to improve dysphagia, and therefore, facilitate easier passage of food and liquid for digestion. Ongoing weight gain, beyond that related to achalasia, may be an unintended consequence of treatment.

Appropriate weight gain after successful achalasia treatment has not been widely studied and data are even more limited examining changes in weight in patients undergoing POEM. A retrospective US study examined BMI and malnutrition risk in 179 patients with achalasia [5]. Of the only 31 patients for whom follow-up data including BMI were available, seven (22.6\%) underwent POEM. While no specific changes in weight were presented for POEM patients, overall, $48.3 \%$ of patients increased their BMI by at least one unit, with $66.7 \%$ considered to be overweight or obese prior to treatment.

Another part-retrospective, part-prospective Japanese study of 72 patients analyzed changes in weight before and 3 months after POEM [9]. Prospective assessment of body composition in 10 patients was performed using dual bioelectrical impedance analysis (BIA). The mean pre-POEM BMI was 20.2, with $27.8 \%$ of patients underweight. Patients were only stratified into underweight and not underweight categories. Among the underweight patients, the mean weight gain at 3 months was $2.9 \mathrm{~kg}$; it was $2 \mathrm{~kg}$ for the not underweight group. It was noted that $25 \%$ of patients had weight gain $\geq 4 \mathrm{~kg}$; these patients had a mean pre-POEM BMI of 19.9. There were significant increases in skeletal muscle mass index and visceral and subcutaneous fat areas in the 10 patients who underwent dual BIA. The modest changes in weight may be related to the short follow-up period; in contrast, our median follow-up period was 15 months. In addition, differences in baseline BMI characteristics would be in keeping with the expected differences due to Eastern compared to Western populations. Only $8.7 \%$ of the patients in our study were underweight at baseline.

Our results are somewhat surprising because a large proportion of the patients - up to $38.5 \%$ in the pre-obese category moved up an entire BMI category on follow-up. Although there 
- Table 2 Patient characteristics according to BMI category.

\begin{tabular}{|c|c|c|c|c|c|}
\hline & \multicolumn{4}{|c|}{ Pre-POEM BMI category } & \\
\hline & Underweight & Normal & Pre-obese & Obese & \\
\hline Count & $6(8 \%)$ & $24(31 \%)$ & $26(34 \%)$ & $21(27 \%)$ & \\
\hline Males & $3(50 \%)$ & $14(58 \%)$ & $16(62 \%)$ & $9(43 \%)$ & $P=0.60$ \\
\hline Age (years) & $30.5(16.8-49.8)$ & $43(30.3-70.5)$ & $60.5(40.8-66.3)$ & $60(47-68.5)$ & $P=0.20$ \\
\hline \multicolumn{5}{|l|}{ Achalasia (Chicago Classification) } & \multirow[b]{5}{*}{$P=0.49$} \\
\hline - Type 1 & $0(0 \%)$ & $6(25 \%)$ & $3(12 \%)$ & $5(24 \%)$ & \\
\hline - Type 2 & $5(83 \%)$ & $11(46 \%)$ & $16(62 \%)$ & $7(33 \%)$ & \\
\hline - Type 3 & $1(17 \%)$ & $6(25 \%)$ & $7(27 \%)$ & $8(38 \%)$ & \\
\hline - Other & $0(0 \%)$ & $1(4 \%)$ & $0(0 \%)$ & $1(5 \%)$ & \\
\hline \multicolumn{6}{|l|}{ Metabolic comorbidities } \\
\hline - Diabetes & $0(0 \%)$ & $3(13 \%)$ & $4(15 \%)$ & $3(14 \%)$ & $P=0.79$ \\
\hline - Dyslipidemia & $0(0 \%)$ & $5(21 \%)$ & $5(19 \%)$ & $8(38 \%)$ & $P=0.20$ \\
\hline - Hypertension & $1(17 \%)$ & $6(25 \%)$ & $9(35 \%)$ & $11(52 \%)$ & $P=0.19$ \\
\hline \multicolumn{5}{|l|}{ Comorbidity burden } & \multirow[b]{5}{*}{$P=0.35$} \\
\hline - None of above & $5(83 \%)$ & $16(67 \%)$ & $16(62 \%)$ & $8(38 \%)$ & \\
\hline - 1 of above & $1(17 \%)$ & $4(17 \%)$ & $5(19 \%)$ & $6(29 \%)$ & \\
\hline - 2 of above & $0(0 \%)$ & $2(8 \%)$ & $2(8 \%)$ & $5(24 \%)$ & \\
\hline - 3 of above & $0(0 \%)$ & $2(8 \%)$ & $3(12 \%)$ & $2(10 \%)$ & \\
\hline Duration of symptoms (months) & $55(16.5-189)$ & $108(30.5-120)$ & $42(12-120)$ & $60(36-134.5)$ & $P=0.32$ \\
\hline Pre-procedure Eckardt & $8.5(6-10.5)$ & $8(6-9)$ & $7.5(6-9)$ & $6(5.5-7.5)$ & $P=0.08$ \\
\hline Myotomy length (cm) & $16.5(12.8-18.5)$ & $16.5(13-20)$ & $17.5(15-20)$ & $17(13.5-19)$ & $P=0.97$ \\
\hline Post-procedure Eckardt & $0(0-1)$ & $1(1-2)$ & $1(0-2)$ & $1(0.5-2)$ & $P=0.48$ \\
\hline Clinical success & $6(100 \%)$ & $21(88 \%)$ & $25(96 \%)$ & $21(100 \%)$ & $P=0.24$ \\
\hline post-POEM reflux (Lyon Consensus) & $3(50 \%)$ & $7(29 \%)$ & $6(23 \%)$ & $3(14 \%)$ & $P=0.33$ \\
\hline
\end{tabular}

Table 3 BMI post-POEM.

\begin{tabular}{|l|c|c|c|c|}
\hline \multicolumn{7}{|c|}{ Pre-POEM BMI category } \\
\hline & Underweight & Normal & Pre-obese & Obese \\
\hline Pre-BMI & $18.0(17-18)$ & $21.4(20.2-23)$ & $27.0(25.4-28.1)$ & $35.9(33.2-37.2)$ \\
\hline Post BMI & $20.7(18.3-25.8)$ & $23.5(21.4-25.9)$ & $28.5(27-31.6)$ & $36.7(33.4-42.4)$ \\
\hline Difference in BMI & $2.7(1.1-8.5)$ & $1.7(0.8-4.1)$ & $2(0-4.6)$ & $0.9(-0.65-3.8)$ \\
\hline Follow-up duration (Months) & $16.5(11.8-26.8)$ & $16.5(9.3-22)$ & $14.5(10.8-25)$ & $18(12-33.5)$ \\
\hline $\begin{array}{l}\text { Continuous data analyzed with Mood's Median Test. } \\
\text { BMI, body mass index; POEM, peroral endoscopic myotomy. }\end{array}$ & & & $P=0.91$ \\
\hline
\end{tabular}


BMI change in underweight category

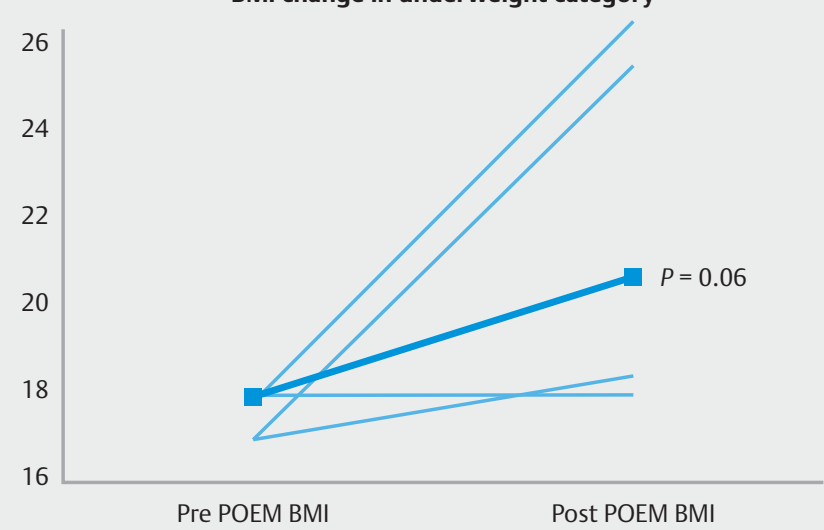

BMI change in pre-obesity category

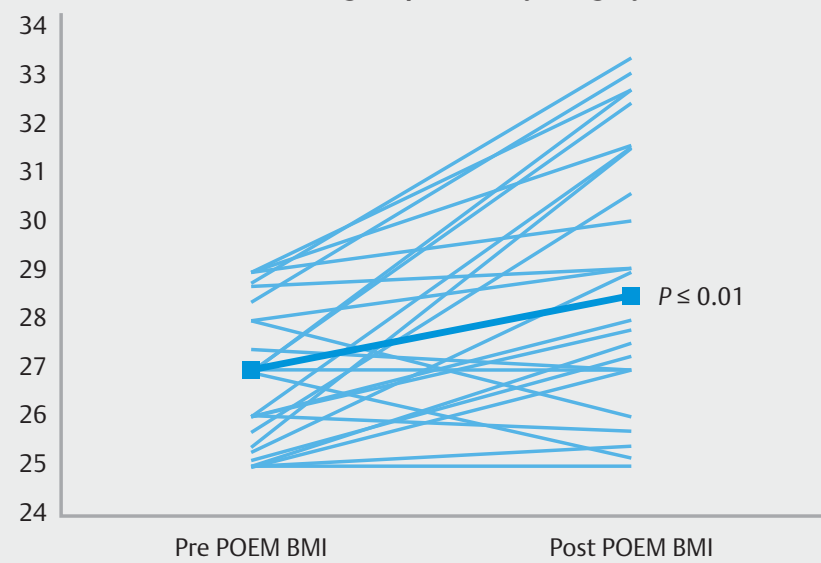

BMI change in normal weight category

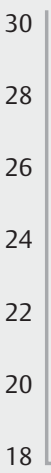

30
28
26
24
22
20
18

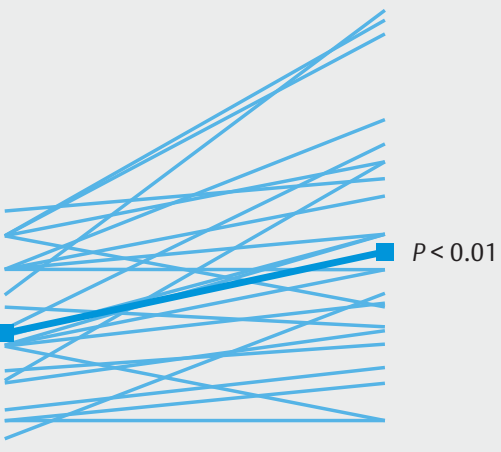

Pre POEM BMI

Post POEM BMI

BMI change in obese category

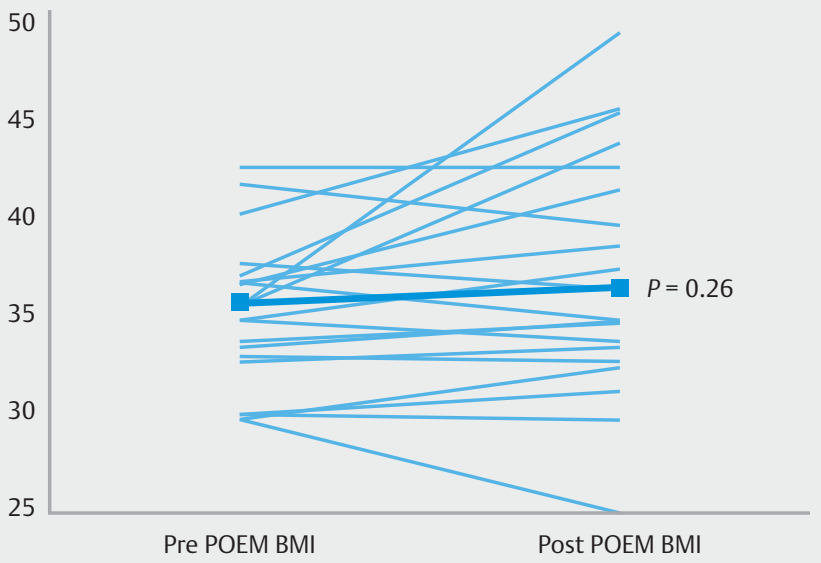

- Fig. 1 Change in BMI by BMI category.

$\checkmark$ Table 4 Changes in BMI category post-POEM.

\begin{tabular}{|c|c|c|c|c|c|}
\hline \multirow[b]{2}{*}{ Post-POEM BMI category } & \multicolumn{4}{|c|}{ Pre-POEM BMI category } & \multirow[b]{2}{*}{ Total } \\
\hline & Underweight & Normal & Pre-Obese & Obese & \\
\hline Underweight & 2 & 0 & 0 & 0 & 2 \\
\hline Normal & 2 & 15 & 0 & 0 & 17 \\
\hline Pre-obese & 2 & 8 & 16 & 1 & 27 \\
\hline Class I obesity & 0 & 1 & 10 & 7 & 18 \\
\hline Class II obesity & 0 & 0 & 0 & 6 & 6 \\
\hline Class III obesity & 0 & 0 & 0 & 7 & 7 \\
\hline Total & 6 & 24 & 26 & 21 & \\
\hline
\end{tabular}

BMI, body mass index; POEM, peroral endoscopic myotomy.

is no consensus about to what extent recovery of pre-morbid weight loss is healthy, but we speculate that moving up a BMI category, especially in those that are already pre-obese or obese, may be excessive. These changes appear to be sustained because our follow-up was a median of 15 months, which ex- tends beyond increases in weight linked to dysphagia resolution immediately post-procedure. Indeed, no patients with baseline pre-obesity or obesity moved into a normal BMI range post-POEM. In general, pre-obesity and obesity have been strongly associated with increased metabolic syndrome and 
cardiovascular [10] and cancer [11] morbidity and mortality, and with one in five of our patients at baseline already having at least one metabolic risk factor, weight gain in our cohort might confer an unintended health risk. Future prospective studies are required to assess for factors and characteristics that are related to weight gain post-POEM and to examine the consequences of such weight gain.

Recent data suggest that achalasia is more prevalent in the overweight or obese population [5]. If our findings are replicated across other Western centers, consideration should be given to introducing pre-POEM education regarding the potential for significant weight gain after POEM. This may involve a multidisciplinary approach, with dieticians and metabolic physicians providing both pre-procedural counseling and also follow-up and support in the months following the procedure. If individuals at high risk of excessive weight gain are identified, a specific diet and exercise program may be required to ensure that weight gain is tempered and controlled. In extreme cases, it may be reasonable to delay POEM until patients achieve a more favorable BMI and metabolic profile pre-procedurally. Alternatively, a combined procedure for achalasia and weight loss may be required, such as POEM and bariatric surgery. Further study is required in this area before clear recommendations can be made.

There are a few limitations to our study. The findings are from a single North American center, and therefore, generalizability cannot be guaranteed. Of note, however, our institution is a nationwide referral center for POEM. Fifteen patients were excluded due to no follow-up BMI being available. The study's retrospective nature prevents detailed analysis regarding weight and other important indices, such as waist circumference, visceral fat, and nutritional biomarkers. BMI class, although predictive of poor health outcomes such as cardiovascular disease and esophageal cancer risk, is just one parameter that contributes to adequate nutrition and health. Comparison with other treatment modalities for achalasia would also provide interesting data, because significant prolonged weight gain also may occur with surgical myotomy and not be exclusive to POEM.

\section{Conclusions}

POEM is an effective treatment for achalasia and weight gain following POEM is almost universal. According to our study in a North American population, in patients with an elevated BMI pre-POEM, weight gain can be substantial and move them into a pre-obese or obesity category. Recognizing this phenomenon is important and may help shape the routine pre- and post-procedural care for POEM patients.

\section{Competing interests}

Dr. Bechara is a consultant for Olympus, Pentax, and Medtronic.

\section{References}

[1] Sadowski DC, Ackah F, Jiang B et al. Achalasia: incidence, prevalence and survival. A population-based study. Neurogastro Motil 2010; 22: 256-261

[2] Inoue H, Minami H, Kobayashi Y et al. Peroral endoscopic myotomy (POEM) for esophageal achalasia. Endoscopy 2010; 42: 265-271

[3] Akintoye E, Kumar N, Obaitan I et al. Peroral endoscopic myotomy: a meta-analysis. Endoscopy 2016; 48: 1059-1068

[4] Li Q, Wu Q, Zhang X et al. Outcomes of per-oral endoscopic myotomy for treatment of esophageal achalasia with a median follow-up of 49 months. Gastrointest Endosc 2018; 87: 1405-1412

[5] Newberry C, Vajravelu RK, Pickett-Blakely O et al. Achalasia patients are at nutritional risk regardless of presenting weight category. Dig Dis Sci 2018; 63: 1243-1249

[6] Kahrilas PJ, Bredenoord A, Fox M et al. The Chicago Classification of esophageal motility disorders, v3.0. Neurogastro Motil 2015; 27: 160-174

[7] Eckardt VF. Clinical presentations and complications of achalasia. Gastrointest Endosc 2001; 11: 281-292

[8] Prakash Gyawali C, Kahrilas P, Savarino E et al. Modern diagnosis of GERD: The Lyon Consensus. Gut 2018; 67: 1351-1362

[9] Mizusawa T, Sato H, Kamimura $\mathrm{K}$ et al. Change in body composition in patients with achalasia before and after peroral endoscopic myotomy. J Gastroenterol Hepatol 2020; 35: 601-608

[10] Calle EE, Thun MJ, Petrelli JM et al. Body-mass index and mortality in a prospective cohort of U.S. adults. N Engl J Med 1999; 341: 1097-1105

[11] Steele CB, Thomas C, Henley S et al. Vital Signs: Trends in Incidence of Cancers Associated with Overweight and Obesity - United States, 2005-2014. MMWR. Morb Mortal Wkly Rep 2017; 66: 1052-1058 